\title{
Integrity Constraint Checking in Federated Databases ${ }^{*}$
}

\author{
Paul Grefen \\ Department of Computer Science \\ University of Twente \\ 7500 AE Enschede, The Netherlands \\ grefen@cs.utwente.nl
}

\author{
Jennifer Widom \\ Department of Computer Science \\ Stanford University \\ Stanford, CA 94305-2140 USA \\ widom@cs.stanford.edu
}

\begin{abstract}
A federated database is comprised of multiple interconnected databases that cooperate in an autonomous fashion. Global integrity constraints are very useful in federated databases, but the lack of global queries, global transaction mechanisms, and global concurrency control renders traditional constraint management techniques inapplicable. This paper presents a threefold contribution to integrity constraint checking in federated databases: (1) The problem of constraint checking in a federated database environment is clearly formulated. (2) A family of cooperative protocols for constraint checking is presented. (3) The differences across protocols in the family are analyzed with respect to system requirements, properties guaranteed, and costs involved. Thus, we provide a suite of options with protocols for various environments with specific system capabilities and integrity requirements.
\end{abstract}

\section{Introduction}

The integration of multiple database systems is fast becoming one of the most important topics in both the research and commercial database communities. Information servers are being developed that provide integrated access to multiple data sources. Legacy database systems are being coupled to form enterprise-wide information systems. Large workflow management applications require routing information through multiple autonomous local systems. The integration of autonomous database systems into loosely-coupled federations requires the development of novel database management techniques specific to these environments.

One important issue in federated database systems is checking integrity constraints over data from multiple sites in the federation. In a federated environment, integrity constraints might specify that replicated information is not contradictory, that certain referential integrity constraints hold, or that some other condition is true over multiple databases. In centralized or tightlycoupled distributed databases, transactions form the cornerstone of integrity constraint checking: Before a transaction commits, it ensures that all constraints are valid. If a constraint is violated, then the transaction may be aborted, the constraint may be corrected automatically, or an error condition may be raised [8].

\footnotetext{
* This work was supported at Stanford by ARPA Contract F33615-93-1-1339
the Anderson Faculty Scholar Fund, and by equipment grants from Digital by the Anderson Faculty Scholar Fund, and by
Equipment Corporation and IBM Corporation.
}

Unfortunately, the lack of inter-site transaction mechanisms in federated databases renders traditional constraint checking mechanisms inapplicable.

This paper addresses the problem of integrity constraint checking in federated databases with a threefold contribution. First, the constraint checking problem is formulated in the specific context of federated databases. In particular, an alternative notion of correctness must be defined, since the transaction-based approach from traditional environments is inappropriate. Then a family of cooperative constraint checking protocols is developed along four protocol "dimensions." Finally, the protocols in the family are analyzed and compared with respect to the requirements of the component database systems, the constraint checking properties guaranteed by the protocols, and the processing and communication costs. By providing a family of protocols, we permit a protocol in the family to be chosen and tailored for the capabilities and requirements of a particular environment.

The structure of the paper is as follows. Below, we first give an outline of related research. Then, Section 2 formalizes the problem of integrity constraint checking in federated databases, describes the context of the work in this paper, and introduces a simple example application. Section 3 presents the family of constraint checking protocols we have developed. Section 4 analyzes the family of protocols by further inspecting the "design space" for protocols and comparing the developed protocols. Finally, we describe how the protocols can be extended to more general architectures. In Section 5 we conclude and discuss future work.

\subsection{Related Work}

Most work addressing the problem of integrity constraint checking in multidatabase environments has considered tightly-coupled distributed databases in which global queries, global transactions, and global concurrency control are present, e.g., $[7,15,18]$. Since these approaches rely on global services that typically are unavailable in federated databases, they are inappropriate for the environment we consider. Note that some approaches focus on relaxing the traditional notion of transaction serializability for constraints in distributed environments, e.g., [3], but some level of locking and global query facilities is still expected.

A few recent papers have addressed the issue of moni- 
toring constraints in loosely-coupled and sometimes heterogeneous database environments. One class of work involves local constraint checking-deriving tests whose success over one database implies the validity of a multidatabase constraint $[1,11,12]$. Local tests optimize the constraint checking process, but they still require a conventional (non-local) method when the local test fails. As will be seen, in this paper we develop protocols that integrate local checking with non-local methods. In [5], a framework and toolkit are described for constraint management in loosely-coupled, highly heterogeneous environments. The focus is on maintaining constraints across systems that have varying capabilities and varying "willingness" to participate in constraint checking protocols, on describing the timing properties associated with constraint checking, and on notions of "conditional consistency" that are weaker than the form of consistency we consider. The issue of maintaining consistency of replicated data across loosely-coupled, semantically heterogeneous databases has been considered in, e.g., $[4,6,17]$. In [4], a method is described that relies on active rules and persistent queues, similar to the simplest case in our family of protocols. Similar issues are addressed in [17], but no specific protocols are provided. In [6], an active rules mechanism is proposed for maintaining replication consistency.

Finally, we note that in the field of distributed (operating) systems there has been considerable work in the area of snapshots and consistent global states, e.g., [2]. Although this work appears highly related to the problem we address, there are two significant differences: (1) The conditions to be evaluated in the distributed system setting are stable, i.e. once a condition becomes valid, it stays valid. This property is not true of database integrity constraints. (2) Protocols for the distributed system setting are designed to obtain some (any) global state, but not to obtain all global states. In contrast, to monitor database constraints it is necessary to monitor all global states, or at least a subset of those states corresponding to consistency "checkpoints".

\section{Preliminaries}

This section presents preliminary material for the remainder of the paper. First, the concepts used in our work are defined formally. Next, we present the basic architecture we consider and discuss implementation aspects of the architecture. Then we describe the class of integrity constraints considered. The section ends with the description of an example application used to motivate our work. Although we cast our work in the context of federations of relational databases, the relational context is introduced primarily for concreteness and clarity. The work easily adapts to other data models.

\subsection{Concepts and Definitions}

Definition 1. A federated relational database system $F$ is a set of $n$ interconnected autonomous database systems $\left\{S_{1}, \ldots, S_{n}\right\}$. Each system $S_{i} \in F$ hosts a local database $D_{i}$ with schema $\mathcal{D}_{i}$. A local database
$D_{i}$ consists of relations $R_{1}^{i}, \ldots, R_{n_{i}}^{i}$ with schemata $\mathcal{R}_{1}^{i}, \ldots, \mathcal{R}_{n_{i}}^{i}$. The set of all relation schemata $\mathcal{R}_{j}^{i}$ in $F$ is called the global database schema $\mathcal{G}$ of $F$.

In the following, we assume a global clock so that we can refer to global times in defining certain concepts. The global clock is used for concept definition only-it is not a requirement of the federated database systems we consider. Also for definitional purposes, we assume that each local database processes its updates in the context of local transactions. However, many of the constraint checking protocols we present are also applicable to local systems that do not support transactions.

Definition 2. The user-observable state of a relation $R_{j}^{i}$ at global time $t$ is the state of $R_{j}^{i}$ reflecting all and only those local update transactions committed before $t$ at site $S_{i}$. The global state $G$ of a global database schema $\mathcal{G}$ at global time $t$ is the set of user-observable states of all relations in $\mathcal{G}$ at global time $t$.

Due to the lack of global transactions in a federated environment, it can be difficult or impossible to observe the state of a global database at a single global time. If an application or protocol does not read local states simultaneously, it may observe a global state that has never actually existed. We call such an observed state a phantom state.

Definition 3. A phantom state $\Phi$ of a global database schema $\mathcal{G}$ observed by application $A$ at time $t_{2}$ as a consequence of a request (or set of requests) by $A$ at time $t_{1}$ is a set of states of all relations in $\mathcal{G}$ such that there exists no $t_{3}$ where $t_{1}<t_{3}<t_{2}$ and the global state of $\mathcal{G}$ at time $t_{3}$ is $\Phi$.

Next, we turn to the definition of global integrity constraints.

Definition 4. A global integrity constraint $I$ is a boolean expression over a global database schema $\mathcal{G}$, i.e., a function $I: \mathcal{G} \rightarrow\{$ true, false $\}$. A global integrity constraint cannot be expressed over a local database schema $\mathcal{D}_{i} \in \mathcal{G}$. A constraint checking protocol for $I$ is an algorithm for evaluating $I$.

In centralized database systems or tightly-coupled distributed database systems, the transitions between database states are determined by transactions. Consequently, integrity constraints generally are required to hold in the states immediately preceding and following each transaction. Since federated environments consist of multiple, autonomous database systems lacking global transactions, we must rely on other concepts to determine the global database states that should satisfy the integrity constraints. We define a notion of global states in which the federated system is "at rest." These quiescent states correspond roughly to the before and after transaction states in traditional database systems, and are the states in which we want to ensure that integrity constraints hold. 
Definition 5. A federated database system $F$ is in a quiescent state at time $t$ if all local update transactions submitted before $t$ have committed, and all constraint checking protocols triggered by any updates before $t$ have completed.

Note that, similar to the before and after transaction states in traditional systems, quiescent states may not physically exist. However, the logical notion of such states is appropriate for defining the correctness of integrity constraint checking. Two important properties of constraint checking protocols are defined with respect to quiescent states: safety and accuracy.

Definition 6. Consider a global database schema $\mathcal{G}$ and a global integrity constraint $I$ over $\mathcal{G}$. A constraint checking protocol for $I$ is safe if the transition from any quiescent global database state $G_{0} \in \mathcal{G}$ that satisfies $I$ to any other quiescent state $G_{1} \in \mathcal{G}$ that does not satisfy $I$ always results in the protocol raising an alarm. ${ }^{1}$ A constraint checking protocol for $I$ is accurate if, after any quiescent global database state $G_{0}$ at time $t_{0}$, a protocol-generated alarm at time $t_{2}$ implies the existence of a global database state $G_{1}$ at time $t_{1}$ such that $t_{0}<t_{1} \leq t_{2}$ and $G_{1}$ does not satisfy $I$.

That is, a protocol is safe if it detects every transition to a quiescent state in which the constraint becomes violated. We assume that safety is required of any constraint checking protocol that will be useful in practice. A safe protocol may be "pessimistic," however, in the sense that it raises too many alarms. A protocol is accurate if, whenever an alarm is raised, there is indeed a state in which the constraint is violated. Although accuracy is a desirable feature of a constraint checking protocol, it is not always necessary. Our last definition involves the representation of database updates.

Definition 7. Consider a relation $R$ in a local database system of a federation. When $R$ is modified, we use $\Delta R$ to denote all modified tuples (inserted, deleted, or updated), $\Delta^{+} R$ to denote all new tuple values (inserts and after-images of updates), $\Delta^{-} R$ to denote all old tuple values (deletes and before-images of updates), and $\Delta^{0} R$ to denote all unmodified tuples. We refer to all forms of $\Delta$ 's as delta sets. We assume that delta sets correspond to the modifications performed by a single local transaction, however other update granularities can be used without affecting our protocols.

\subsection{Basic Architecture}

Figure 1 depicts the basic architecture we address for integrity constraint checking in federated databases. To develop our protocols we consider the restricted case of two databases, each containing a single relation. However, our protocols can be generalized to handle constraints over more than two sites-see Section 4.2 for a

\footnotetext{
${ }^{1}$ In this paper we do not consider the reaction to constraint violations. Rather, we focus on the detection of constraint violations, and we say that when a protocol detects a constraint violation it raises an "alarm."
}

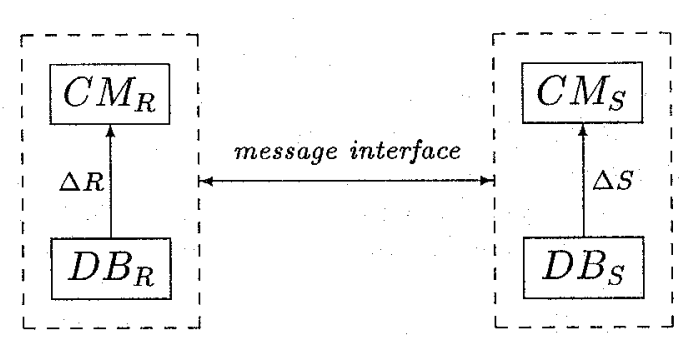

Figure 1: Basic architecture

discussion. Generalizing to more than one relation per site is straightforward.

In the diagram, $D B_{R}$ and $D B_{S}$ denote two local database systems managing relations $R$ and $S$, respectively. Connected to the local database systems are constraint managers $C M_{R}$ and $C M_{S}$. There is one constraint manager for each local database handling the global constraints that may be invalidated by operations on its corresponding database. Consequently, each global constraint is replicated at every site containing data involved in the constraint. We assume that any local constraints are managed within the appropriate database system (see, e.g., [8]).

The constraint manager is notified of all changes $(\Delta ' s)$ to the local database that may violate a constraint. For convenience, we may assume that delta notifications are sent at the end of each local update transaction; however, any granularity of delta notification can be handled by our protocols. Of particular importance is that we do not generally assume that delta notifications or subsequent constraint checking protocols occur as part of a local transaction, and constraint managers need not be tightly coupled with their corresponding database systems. The database systems in the federation are autonomous, in that global queries, global transactions, and global concurrency control mechanisms are not available. A message-passing interface connects the two sites. This interface allows the constraint managers to exchange messages in a cooperative fashion, and may allow a constraint manager to send requests directly to the remote database system.

An essential aspect of implementing the above architecture is supporting the delta notification mechanism by which database systems in a federation communicate with their respective constraint managers. Several implementation schemes can be used to realize this mechanism. Database rules following the eventcondition-action (ECA) paradigm are currently supported by many active database systems [19]. These rules can be used to implement the delta notification mechanism in a straightforward way. For database systems that do not include an active rule manager, passive rules can be used-passive rules are processed by a transaction modification component built on top of a conventional database system [9, 14]. Database triggers as supported by many recent commercial database systems can be used in a similar way to rules. Finally, in cases where no triggering mechanism is available at 


\begin{tabular}{|l|l|}
\hline system & relation \\
\hline$S_{A}$ & Patients \\
$S_{B}$ & Patients \\
$S_{A}(S S N$, Name, Physician $)$ \\
$S$ & Physicians PhN, Name $)$ \\
\hline
\end{tabular}

Table 1: Example database relations

the database system level (e.g., in strictly closed legacy systems), delta notification must be handled by the constraint manager using a polling mechanism. In this approach, the constraint manager polls the local database system at a certain frequency to detect when changes have occurred. Certainly this approach is the least desirable from both performance and architecture standpoints, but is necessary in the case of closed systems with no notification mechanism.

\subsection{Integrity Constraints}

In this paper we consider constraints over two relations, $R$ and $S$. In particular, we consider constraints that can be evaluated incrementally $[7,16]$. By incremental, we mean that if the constraint is valid initially, and if one of the relations is changed - relation $R$, say-then the constraint can be reevaluated by considering only the changes to $R(\Delta R)$ and the relation $S$. This class of constraints includes many of the most common constraint types, such as referential integrity and mutual exclusion. See the technical report version of this paper [10] for a complete definition of the class of constraints considered. The protocols we present can be extended in a straightforward way to constraints over more than two relations; the two-relation restriction is adopted for clarity and brevity only.

We assume that constraints are expressed as queries, where the constraint is satisfied iff the query result is empty. This is a common and convenient formulation $[8,11,10]$, equivalent to expressing constraints as logical formulae. Hence, we denote a constraint $C$ over relations $R$ and $S$ as a query $Q(R, S)$. A query to check $C$ incrementally with respect to changes on $R$ is denoted $Q^{\prime}(\Delta R, S)$, where again the constraint is satisfied iff the query result is empty [16]. $Q^{\prime}(R, \Delta S)$ is similar. Below, we introduce an example application illustrating this notation.

Suppose we have two hospitals, $H_{A}$ and $H_{B}$, each having its own database system, $S_{A}$ and $S_{B}$ respectively. Because the hospitals cooperate by sharing physicians, the two database systems are connected into a federation, the simplified schema of which is shown in Table 1. Both hospitals keep a local record of their patients. To avoid duplication, all physicians are registered at hospital $H_{A}$. The hospitals enforce a rule that each patient can be registered at only one hospital. In addition, all patients must have a registered physician. These rules lead to the following three integrity constraints, expressed as relational algebra queries:

$C_{1}:$ Patients $_{A} \bowtie_{S S N=S S N}$ Patients $_{B}$

$C_{2}: \Pi_{P_{h y s i c i a n}}$ Patients $_{A}-\Pi_{p_{h N}}$ Physicians

$C_{3}: \Pi_{P h y s i c i a n}$ Patients $s_{B}-\Pi_{P h N}$ Physicians

Constraints $C_{1}$ and $C_{3}$ are global constraints, since they involve relations at two different sites. Constraint $C_{2}$ is a local constraint, so it can be enforced at its local site $\left(S_{A}\right)$ using standard methods. Constraints $C_{1}$ and $C_{3}$ can each be "factored" into two incremental global constraints, dealing with relevant updates to each of the two relations:

$$
\begin{aligned}
& C_{1}^{A}: \Delta^{+} \text {Patients }_{A} \bowtie_{S S N=S S N} \text { Patients }_{B} \\
& C_{1}^{B}: \text { Patients } \bowtie_{S S N=S S N} \Delta^{+} \text {Patients }{ }_{B} \\
& C_{3}^{B}: \Pi_{P h y s i c i a n} \Delta^{+} \text {Patients } s_{B}-\Pi_{P h N} \text { Physicians } \\
& C_{3}^{A}: \Pi_{\text {Physician Patients }} \cap \Pi_{P h N} \Delta^{-} \text {Physicians }
\end{aligned}
$$

\section{The Family of Protocols}

In this section, we develop a family of cooperative constraint checking protocols suitable for federated databases. The members of this family have different requirements, different properties, and different performance characteristics. The "root" of the family is a very simple protocol, described in Section 3.1. In Sections $3.2-3.5$ we enhance the simple protocol to obtain more useful protocols. In designing the protocols, we are especially interested in the safety and accuracy properties as defined in Section 2.1. The root of the protocol family is safe, and so are all of the protocols derived from it. To obtain accuracy, we enhance the root protocol along the dimensions of timestamping mechanisms and local transaction mechanisms. Timestamping mechanisms annotate delta sets and query results with global timestamps, enabling the algorithm to detect when phantom states may have been used in constraint evaluation. In contrast, local transaction mechanisms can be used to prevent the evaluation of constraints over phantom states.

In addition to safety and accuracy, we also are interested in the performance of the protocols, and in ensuring that the local databases remain as autonomous as possible. For these purposes, we enhance the protocols along the dimensions of change logging mechanisms and local test mechanisms. Change logging mechanisms accumulate updates in special purpose data sets so that database updating and constraint checking need not occur at the same granularity. Local test mechanisms check global constraints by accessing local data only (whenever possible), thus avoiding any kind of global coordination.

These four dimensions delineate a "design space" for constraint checking protocols. In the remainder of this section, the various protocols are developed, beginning with the simple protocol at the "origin" of the design space. For the specification of a protocol, we use a table that contains a set of sequentially occurring steps. Each step is performed by an actor - a database system or a constraint manager. Associated with each step is an action. Actions vary from simple query evaluations or messaging commands to more complex behavior. For the illustration of a protocol, we use a figure showing how the protocol behaves within the context of the basic architecture introduced earlier in Figure 1. 


\begin{tabular}{|c|cl|}
\hline 1 & $D B_{R}$ & send $\triangle \overline{R \text { to } C M_{R}}$ \\
2 & $C M_{R}$ & receive $\Delta R$ from $D B_{R}$ \\
3 & & send $Q(\Delta R, S)$ to $D B_{S}$ \\
4 & $D B_{S}$ & receive $Q(\Delta R, S)$ from $C M_{R}$ \\
5 & & evaluate $Q(\Delta R, S)$ \\
6 & & send query result to $C M_{R}$ \\
7 & $C M_{R}$ & receive query result from $D B_{S}$ \\
8 & & raise alarm if query result is non-empty
\end{tabular}

Table 2: DRQ Protocol

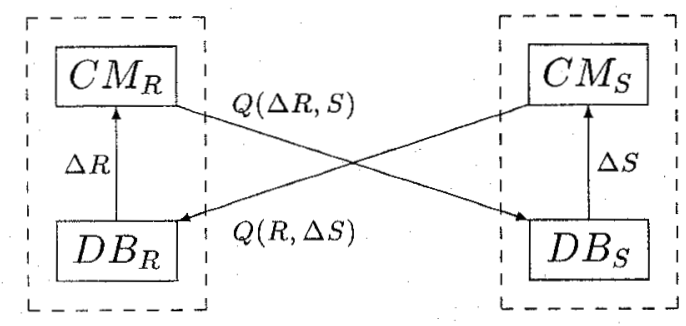

Figure 2: DRQ Protocol

\subsection{DRQ and IRQ Protocols}

The Direct Remote Query Protocol (DRQ) is specified in Table 2 and depicted in Figure 2. Table 2 specifies how the protocol responds to a delta notification for relation $R$. A symmetric protocol is used to handle updates to $S$. Protocols handling updates to $R$ and to $S$ may run concurrently. We will assume that multiple updates to $R$ or to $S$ are handled sequentially, although this assumption is not strictly necessary. Figure 2 illustrates the protocol for both updates to $R$ and to $S$.

The DRQ protocol is very simple: When the constraint manager is notified of an update, it sends the appropriate incremental query for evaluation at the other site. Delta sets are usually small enough that the actual data can be transmitted in a straightforward way. For example, $Q(\triangle R, S)$ may by a query over $S$ with the values from $\Delta R$ "plugged in." If the query result is nonempty, then the constraint manager raises an alarm. The DRQ protocol always detects when a constraint is violated, i.e., it is safe. Unfortunately, DRQ can easily produce "false alarms," i.e., it is inaccurate.

To show that the DRQ protocol is safe, we show that starting from a quiescent consistent global state $D_{0}$ at time $t_{0}$, a quiescent inconsistent global state $D_{n}$ at time $t_{n}$ cannot be reached without an alarm being raised: (1) At least one update must have occurred between times $t_{0}$ and $t_{n}$ to reach an inconsistent quiescent state $D_{n}$ from consistent state $D_{0}$. (2) Assume that update $u_{1}$ occurring at time $t_{1}, t_{0}<t_{1}<t_{n}$, is the last such update producing an inconsistent state. (3) Update $u_{1}$ triggers a constraint checking process that does not raise an alarm, so there exists a time $t_{2}, t_{1}<t_{2}<t_{n}$, at which the state is consistent. (4) Since the state at $t_{n}$ is inconsistent, there must be an update $u_{2}$ at time $t_{3}$, $t_{2}<t_{3}<t_{n}$, that causes the inconsistent state. This contradicts the fact that $u_{1}$ is the last update invalidating the constraint.

The inaccuracy of DRQ can be easily be shown by a

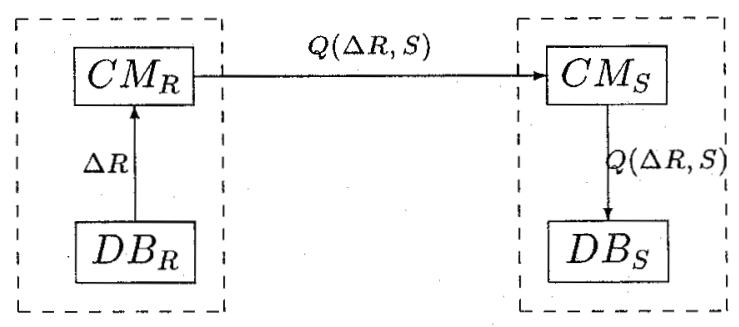

Figure 3: IRQ Protocol

counterexample in which an alarm is raised but there is never an inconsistent global state [10]. Inaccuracy is due to the fact that the DRQ protocol may evaluate the constraint over a phantom state, since relations $R$ and $S$ are accessed at different times. We can solve the problem in two ways, by detection or by prevention: (1) We can use timestamping information to detect possible evaluation over phantom states. (2) We can exploit local transaction mechanisms to prevent evaluation over phantom states. The first solution is explored in Section 3.2, the second solution in Section 3.3. First, a slight variation on the DRQ protocol is presented.

The DRQ protocol relies on the capability of each database system to process queries issued from a remote constraint manager. If remote query services are unavailable, then we can use a variation on the DRQ protocol that uses peer-to-peer communication between the constraint managers. This Indirect Remote Query Protocol (IRQ) is illustrated in Figure 3. For clarity, the figure (and all protocol figures to follow) only shows the case where $R$ is updated. The case where $S$ is updated is symmetric in all protocols. Like the DRQ protocol, the IRQ protocol is safe but inaccurate.

\subsection{TRQ and SRQ Protocols}

The DRQ and IRQ protocols lack the accuracy property because they cannot distinguish true global states from phantom states. To overcome this problem, we first enhance the IRQ protocol with a timestamping technique. This technique allows the constraint manager to detect when a phantom state may have been used for query evaluation, and to reevaluate queries when this happens. To make the protocol more efficient, when reevaluation is necessary it is performed with a cumulative delta set, i.e., a delta set combining multiple delta notifications from a single site with net effect semantics.

The Timestamped Remote Query Protocol (TRQ) is specified in Table 3 and depicted in Figure 4 . In the figure and the table, the $t_{i}$ 's denote timestamps attached to messages, and $\Delta R @ t$ and $\Delta S @ t$ denote notifications of updates occurring at time $t$. Assume for now that a synchronous global clock is used to generate timestamps; we discuss later how this assumption can be relaxed. The TRQ protocol requires that the interface between each constraint manager and its database system is order preserving. In particular, a query result sent to the constraint manager must follow the notification of a relevant update if that update was performed 


\begin{tabular}{|r|cl|}
\hline 1 & $D B_{R}$ & send $\left\langle\Delta \bar{R}, t_{1}\right\rangle$ to $C M_{R}$ \\
2 & $C M_{R}$ & receive $\left\langle\Delta R, t_{1}\right\rangle$ from $D B_{R}$ \\
3 & & send $\left\langle Q(\Delta R, S), t_{1}\right\rangle$ to $C M_{S}$ \\
4 & $C M_{S}$ & receive $\left\langle Q(\Delta R, S), t_{1}\right\rangle$ from $C M_{R}$ \\
5 & & submit $Q(\Delta R, S)$ to $D B_{S}$ \\
6 & $D B_{S}$ & receive $Q(\Delta R, S)$ from $C M_{S}$ \\
7 & & evaluate $Q(\Delta R, S)$ \\
8 & & send query result to $C M_{S}$ \\
9 & $C M_{S}$ & receive query result from $D B_{S}$ at $t_{2}$ \\
10 & & IF $\left(\exists t_{3}\right)\left(\Delta S @ t_{3} \wedge t_{1}<t_{3}<t_{2}\right)$ \\
& & THEN send $\left\langle q u e r y r e s u l t\right.$, busy, $\left.t_{3}\right\rangle$ to $C M_{R}$ \\
& & ELSE send $\langle q u e r y r e s u l t, q u i e t\rangle$ to $C M_{R}$ \\
11 & $C M_{R}$ & receive query result and status from $C M_{S}$ \\
12 & & IF status=quiet OR $\left(\nexists t_{4}\right)\left(\Delta R @ t_{4} \wedge t_{1}<t_{4}<t_{3}\right)$ \\
& & THEN raise alarm if query result is non-empty \\
& & ELSE restart from step 2 with cumulative $\Delta R$
\end{tabular}

Table 3: TRQ Protocol

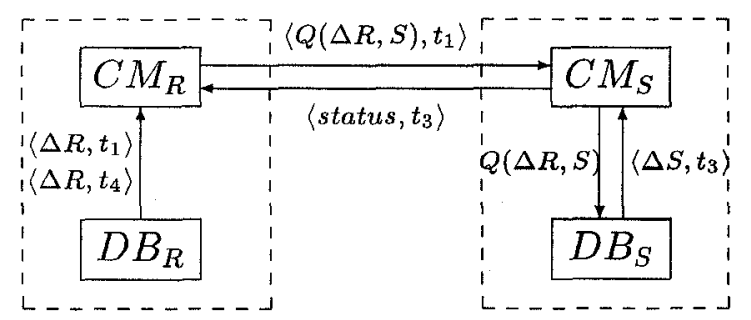

Figure 4: TRQ Protocol

before the query was evaluated.

The TRQ protocol behaves as follows. When constraint manager $C M_{R}$ is notified of an update to $R$, it sends the appropriate incremental query $Q$ for evaluation to constraint manager $C M_{S}$, along with a timestamp for the update. $C M_{S}$ requests evaluation of query $Q$ at database $D B_{S}$. If an update occurs to $S$ between the time of $R$ 's update and the evaluation of $Q$, then $C M_{S}$ returns a busy status to $C M_{R}$ (rather than a quiet status) along with the query answer. Consider what happens when $C M_{R}$ receives the answer. If the return status is quiet then there have been no relevant updates to $S$ and the query has been evaluated over a true global state. If the return status is busy then there have been relevant updates to $S$. However, if $R$ has not been updated further, then the query still has been evaluated over a true global state. In the case where there have been further updates to $R$, then the query may have been evaluated over a phantom state, and it must be reevaluated. Reevaluation takes place by restarting the protocol; for efficiency, the original and new updates to $R$ are combined before restarting. Note that, provided that updates to $R$ and $S$ eventually cease, termination of the protocol is guaranteed since protocol restarts are triggered only by new updates.

Safety of the TRQ protocol follows the same line of reasoning as safety for the DRQ protocol. We show that the TRQ protocol is accurate by arguing that every successful (i.e., not restarted) query evaluation is performed in a true global state: (1) After an update $\Delta R$ at time $t_{1}, t_{1}$ is the first "candidate" global state for evaluation of $Q$. If evaluation does not produce a

\begin{tabular}{|r|cl|}
\hline 1 & $D B_{R}$ & send $\Delta R$ within $t_{\Delta}$ to $C M_{R}$ \\
2 & $C M_{R}$ & receive $\Delta R$ from $D B_{R}$ at $t_{1}$ \\
3 & & send $\left\langle Q(\Delta R, S), t_{1}-t_{\Delta}\right\rangle$ to $C M_{S}$ \\
4 & $C M_{S}$ & receive $\left\langle Q(\Delta R, S), t_{1}-t_{\Delta}\right\rangle$ from $C M_{R}$ \\
5 & & submit $Q(\Delta R, S)$ to $D B_{S}$ \\
6 & $D B_{S}$ & receive $Q(\Delta R, S)$ from $C M_{S}$ \\
7 & & evaluate $Q(\Delta R, S)$ \\
8 & & send query result to $C M_{S}$ \\
9 & $C M_{S}$ & receive query result from $D B_{S}$ at $t_{2}$ \\
10 & & IF $\left(\exists t_{3}\right)\left(\Delta S @ t_{3} \wedge t_{1}-t_{\Delta}<t_{3}<t_{2}\right)$ \\
& THEN send $\left\langle q u e r y r e s u l t\right.$, busy, $\left.t_{3}\right\rangle$ to $C M_{R}$ \\
& & ELSE send $\langle$ queryresult, quiet $\rangle$ to $C M_{R}$ \\
11 & $C M_{R}$ & receive query result and status from $C M_{R}$ \\
12 & & IF status $=q u i e t$ OR \\
& ( $\left.\nexists t_{4}\right)\left(\Delta R @ t_{4} \wedge t_{1}<t_{4} \wedge t_{4}-t_{\Delta}<t_{3}\right)$ \\
& THEN raise alarm if query result is non-empty \\
& ELSE restart from step 2 with cumulative $\Delta R$ \\
\hline
\end{tabular}

Table 4: SRQ Protocol

busy status, then $Q$ has been evaluated in the state occurring at time $t_{1}$. (2) If $C M_{S}$ identifies a $\Delta S$ at time $t_{3}$ with $t_{1}<t_{3}<t_{2}$, then $t_{3}$ is taken as the next candidate global state. If there have been no further updates to $R$ before $t_{3}$, then $Q$ has been evaluated in the state occurring at time $t_{3}$. (3) If $C M_{R}$ identifies a new $\Delta R$ at time $t_{4}$ with $t_{1}<t_{4}<t_{3}$, then the process is restarted with $t_{3}$ as the next candidate global state.

The TRQ protocol assumes that the database systems can generate timestamps. A similar protocol can be used for the situation in which the database systems cannot generate timestamps, but the constraint managers can. To provide the accuracy property when timestamps are generated by the constraint managers, it is necessary to guarantee that delta notifications from database systems are always received within a certain maximum delay $t_{\Delta}$ [5]. For this scenario we provide the Semi-timestamped Remote Query Protocol (SRQ), specified in Table 4. The SRQ protocol is very similar to the TRQ protocol, except the determination of whether a phantom state may have been used must take into account the notification delays $t_{\Delta}$. Like the TRQ protocol, the SRQ protocol is safe and accurate.

Both the TRQ and SRQ protocols require access to a synchronous global clock. However, suppose that instead of a global clock we have a set of local clocks that are synchronized within a maximum drift $t_{D}$. Then the local clocks can be used instead of a global clock by incorporating $t_{D}$ into our protocols, similarly to the way we have incorporated $t_{\Delta}$ to obtain the SRQ protocol from TRQ. In addition, we are investigating whether we can adapt our protocols so that logical clocks $[2,13]$ are sufficient.

\subsection{TRT Protocol}

The TRQ and SRQ protocols add timestamping to the DRQ protocol in order to achieve accuracy. This approach corresponds to one dimension in the protocol design space. The next dimension we consider is transactions. Note that we are not proposing to add global transaction capabilities, but to exploit standard transaction capabilities provided by the local database systems. The Transaction Remote Transaction Protocol 


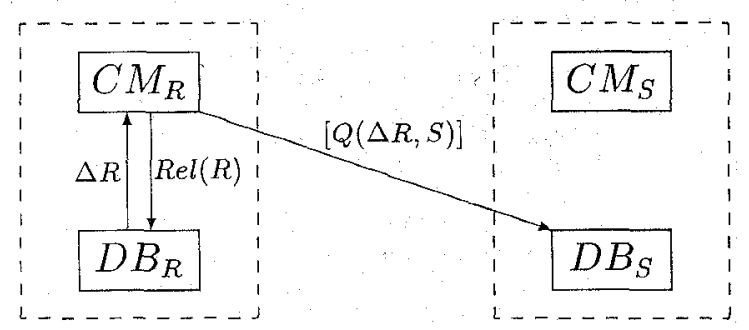

Figure 5: TRT Protocol

(TRT) is depicted in Figure 5. In the figure, the enclosure of a query in square brackets indicates that the query is to be executed within its own local transaction.

The TRT protocol behaves as follows. When $D B_{R}$ performs a delta notification, this notification occurs as part of the transaction $\tau$ that updated $R$, and $\tau$ does not commit until it receives a "release" message from the constraint manager. That is, transaction $\tau$ continues to hold its exclusive lock ( $X$-lock) on $R$ while the protocol runs. This approach requires that the database system is capable of performing a notification and waiting for an acknowledgment, all within a single transaction. This capability is provided by most database systems supporting triggers or active rules [19]; see Section 2.2. Once $C M_{R}$ has received the delta notification, it sends the appropriate query to $D B_{S}$, to be executed within its own transaction $\tau^{\prime}$ at $D B_{S}$. During execution of the query, $\tau^{\prime}$ will hold a shared lock $(S$-lock $)$ on $S$. When the query answer is received by $C M_{R}$, an alarm is raised if the result is non-empty, and the pending transaction $\tau$ at $D B_{R}$ is released.

Safety of the TRT protocol follows the same line of reasoning as for the other protocols. Accuracy of the TRT protocol results from the fact that every query is evaluated over a true global state (with respect to $R$ and $S$ ). To see that only true global states are used, we can think of the bracketed query transaction $\tau^{\prime}$ as being embedded within the pending transaction $\tau$ that generated the delta notification. In this way, the TRT protocol effectively emulates a distributed two-phase locking protocol, ensuring serializability across sites.

Although the TRT protocol is relatively straightforward, and it satisfies both the safety and accuracy properties, it has two significant drawbacks. (1) Constraint manager processing is synchronous with the database system, unlike in the previous protocols. In TRT, the database system must wait for the constraint checking protocol to complete before it can commit its transaction and release its locks. This is a loss of autonomy, since progress on one site is dependent upon progress on the other site. (2) If $R$ and $S$ are updated concurrently then the risk of deadlock is relatively high. The same deadlock cases arise as in centralized databases, but executing a remote transaction in a loosely-coupled environment may be much slower than transaction processing in a centralized environment, thereby increasing the chance of deadlock. These drawbacks are addressed in an extended version of the TRT protocol called MDS,

\begin{tabular}{|c|c|c|}
\hline 1. & $D B_{R}$ & append change to $\Delta \bar{R}$ \\
\hline 2 & & send $\Delta$ notification to $C M_{R}$ \\
\hline 3 & $C M_{R}$ & receive $\Delta$ notification from $D B_{R}$ \\
\hline 4 & & $\begin{array}{l}\text { decide whether to run constraint check; } \\
\text { if not, terminate }\end{array}$ \\
\hline 5 & & open transaction on $D B_{R}$, X-locking $\Delta R$ \\
\hline 6 & & submit $Q(\Delta R)$ to $D B_{R}$ \\
\hline 7 & $D B_{R}$ & receive $Q(\Delta R)$ from $C M_{R}$ \\
\hline 8 & & evaluate $Q(\Delta R)$ \\
\hline 9 & & send query result to $C M_{R}$ \\
\hline 10 & $C M_{R}$ & receive query result from $D B_{R}$ \\
\hline 11 & & send $[Q(\Delta R, S)]$ to $D B_{S}$ \\
\hline 12 & $D B_{S}$ & receive $[Q(\triangle R, S)]$ from $C M_{R}$ \\
\hline 13 & & execute $[Q(\Delta R, S)]$, S-locking $S$ \\
\hline 14 & & commit transaction, releasing locks on $S$. \\
\hline 15 & & send query result to $C M_{R}$ \\
\hline 16 & $C M_{R}$ & receive result from $D B_{S}$ \\
\hline 17 & & raise alarm if query result is non-empty \\
\hline 18 & & submit Delete $(\Delta R)$ to $D B_{R}$ \\
\hline 19 & $D B_{R}$ & perform Delete $(\Delta R)$ \\
\hline 20 & $C M_{R}$ & $\begin{array}{l}\text { commit transaction on } D B_{R}, \\
\text { releasing X-lock on } \Delta R\end{array}$ \\
\hline
\end{tabular}

Table 5: MDS Protocol

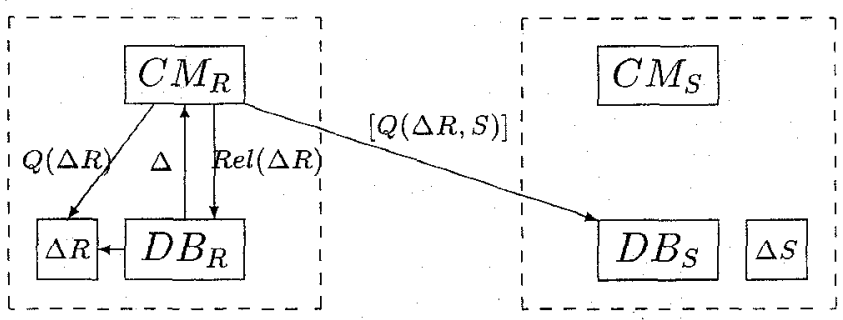

Figure 6: MDS Protocol

presented next. Another improvement to the TRT protocol is to use a local test to avoid the problems caused by: remote transactions, described in Section 3.5.

\subsection{MDS Protocol}

Our next protocol considers the change logging dimension of the protocol design space. In this protocol, relevant changes are "logged" in special relations that we call materialized delta sets. Materialized delta sets allow us to develop a version of the TRT protocol in which constraint checking is decoupled from database execution, thereby addressing the drawbacks associated with the TRT protocol. In addition, materialized delta sets enable variable, application-dependent granularities for constraint checking.

The Materialized Delta Set Protocol (MDS) is specified in Table 5 and depicted in Figure 6 . It behaves as follows. When relation $R$ is updated, $D B_{R}$ appends the update to the materialized delta set $\Delta R$. (Here, $\Delta R$ denotes an accumulated set of changes, not just an individual change or set of changes.) $D B_{R}$ 's append to $\Delta R$ must occur before the local transaction that updates $R$ commits. A notification $\Delta$ is sent to $C M_{R}$, indicating that an update occurred but not providing the actual delta set. When $C M_{R}$ decides to proceed with constraint checking, it obtains an X-lock on $\Delta R$ for the duration of the protocol. $C M_{R}$ then reads $\Delta R$, and proceeds just as in the TRT protocol, executing a query $Q(\Delta R, S)$ within a transaction at the remote site. 


\begin{tabular}{|c|cl}
\hline 1 & $D B_{R}$ & send $\triangle R$ to $C M_{R}$, holding transaction \\
& & X-locking $R$ \\
2 & $C M_{R}$ & receive $\triangle R$ from $D B_{R}$ \\
3 & & submit $Q_{L}(\Delta R, R)$ to $D B_{R}$ \\
4 & $D B_{R}$ & receive $Q_{L}(\Delta R, R)$ from $D B_{R}$ \\
5 & & evaluate $Q_{L}(\Delta R, R)$ \\
6 & & send query result to $C M_{R}$ \\
7 & $C M_{R}$ & receive query result from $D B_{R}$ \\
8 & & IF query result is non-empty \\
& & THEN perform TRT in same transaction \\
\hline
\end{tabular}

Table 6: LTT Protocol

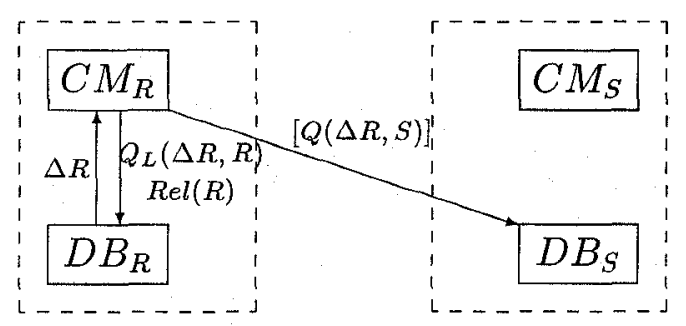

Figure 7: LTT Protocol

After receiving the query result and raising an alarm if appropriate, $C M_{R}$ erases the current contents of materialized delta set $\Delta R$ and releases its X-lock on $\Delta R$.

As in the TRT protocol, accuracy is guaranteed because the protocol emulates distributed two-phase locking. However, unlike in TRT, local access to $R$ is not entirely restricted while the protocol is executing. Local transactions that read $R$ can execute concurrently with the protocol. Concurrent local transactions also can update $R$; however, because the protocol X-locks $\Delta R$, such transactions cannot complete and commit until the protocol has finished. In addition, the risk of deadlock is much lower with MDS than with TRT [10].

\subsection{LTT Protocol}

The last dimension of the protocol design space we explore is the use of local tests. Consider a delta notification $\Delta R$. A local test is a query, $Q_{L}(\Delta R, R)$, such that if the query result is empty, then the result of the global constraint checking query $Q(\Delta R, S)$ is guaranteed to be empty as well $[12,11]$. The test is "local" because it involves $\Delta R$ and $R$ only, and does not require access to $S$. Hence, a local test can be used to avoid remote queries. Unfortunately, local tests are generally conservative, so if a local test fails it becomes necessary to issue a remote query.

The Local Test Transaction Protocol (LTT) is specified in Table 6 and depicted in Figure 7. The protocol is very similar to TRT, except before issuing the remote transaction $Q(\Delta R, S)$, the local test $Q_{L}(\Delta R, R)$ is evaluated. If the local test succeeds, then the protocol terminates successfully with no remote activity. Note that the local test must be evaluated within the same transaction in which the update occurred in order to behave correctly [11], so a materialized delta set approach cannot be applied here. The safety and accuracy of LTT follows from the correctness of local tests $[12,11]$, and from the safety and accuracy of TRT.

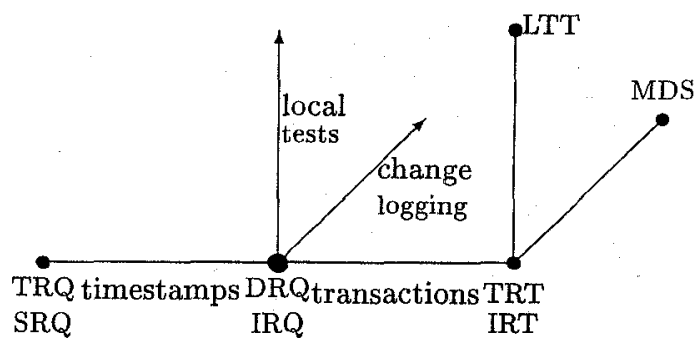

Figure 8: Protocols in the protocol space

\section{Analyzing the Family}

In Section 3 we developed a family of constraint checking protocols along the four dimensions of the protocol design space. In this section we analyze the protocols. First, we consider the protocols in the context of the design space to see how complete the family is. Then we compare the characteristics and costs of the various protocols, and discuss how the protocols can be extended to handle constraints spanning more than two databases.

\subsection{The Protocol Space}

Figure 8 places the protocols developed in Section 3 into their appropriate positions in the four-dimensional protocol design space. Although several combinations of dimensions have been explored, not every possibility has been considered, as four dimensions yield sixteen possible protocols.

We first point out three potentially useful protocols not discussed in Section 3, combining features from our protocols in a relatively straightforward way. Change logging can be used with DRQ or IRQ to produce a Cumulative Remote Query (CRQ) protocol, which accumulates changes in a materialized delta set to allow variable granularities of constraint checking. Timestamps and change logging can be combined to obtain a Timestamped Materialized Delta Set (TMD) protocol, in which materialized delta sets containing timestamps are used in order to allow variable granularities of constraint checking and to keep track of cumulative updates for protocol restarts. Timestamps, transaction mechanisms, and local tests can be combined into a Local Transaction Global Timestamped (LTG) protocol. LTG resembles LTT, except when a local test fails, timestamping is used rather than transactions for the global query (to achieve more autonomy).

Some points in the design space have an inappropriate combination of features: Local tests cannot be used without local transactions. Combining timestamps and transactions is "overkill" in the case where local tests are not used. Finally, the combination of transactions, change logging, and local tests yields rather complex protocols that we have yet to explore.

\subsection{Comparing the Protocols}

The various protocols in the family can be compared with respect to what they require, and what they deliver. The requirements of the protocols concern the 


\begin{tabular}{|l|c|c|c|c|c|c|c|c|}
\hline & \multicolumn{4}{|c|}{ requirements } & \multicolumn{4}{|c|}{ properties } \\
\hline & $\begin{array}{c}\text { rem } \\
\text { qry }\end{array}$ & $\begin{array}{c}\text { loc } \\
\text { tr }\end{array}$ & $\begin{array}{c}\text { ord } \\
\text { pre }\end{array}$ & $\begin{array}{c}\text { glb } \\
\text { cick }\end{array}$ & safe & acc & asyn & flex \\
\hline DRQ & $\mathrm{X}$ & & & & $\mathrm{X}$ & & $\mathrm{X}$ & \\
IRQ & & & & & $\mathrm{X}$ & $\mathrm{X}$ & $\mathrm{X}$ & \\
TRQ & & & $\mathrm{X}$ & $\mathrm{X}$ & $\mathrm{X}$ & $\mathrm{X}$ & $\mathrm{X}$ & \\
SRQ & & & $\mathrm{X}$ & $\mathrm{X}$ & $\mathrm{X}$ & $\mathrm{X}$ & $\mathrm{X}$ & \\
TRT & $\mathrm{X}$ & $\mathrm{X}$ & & & $\mathrm{X}$ & $\mathrm{X}$ & & \\
MDS & $\mathrm{X}$ & $\mathrm{X}$ & & & $\mathrm{X}$ & $\mathrm{X}$ & & $\mathrm{X}$ \\
LTT & $\mathrm{X}$ & $\mathrm{X}$ & & & $\mathrm{X}$ & $\mathrm{X}$ & & \\
\hline
\end{tabular}

Table 7: Requirements and properties of the protocols

functionality of the systems within the federation and the functionality of the interfaces between systems: an interface for executing remote queries, availability of local database transactions, an order-preserving interface between each database system and its corresponding constraint manager, and a global clock. The protocols "deliver" certain properties: safety, accuracy, asynchrony, and flexible granularity for constraint checking. Table 7 summarizes the requirements and properties of the protocols discussed in Section 3.

The requirements and properties discussed so far are static characteristics of the protocols. The protocols also can be compared in terms of their dynamic characteristics, specifically their execution costs. We distinguish three ingredients in the cost of a constraint checking protocol. Local messages are messages between a database system and its constraint manager. We distinguish between notification-only messages and messages that convey data. Remote messages are messages between a constraint manager and a different constraint manager or a remote database system. All remote messages convey data. Database operations are operations performed by a local database system, where an operation can be either a query, a database modification, or a transaction commit. The cost functions for the protocols from Section 3 are shown in Table 8. Costs are given for a single delta notification, i.e., for one local update transaction triggering the protocol.

From the analysis in Table 8 some interesting observations can be made.Although the TRQ protocol has better properties than the IRQ protocol, and TRQ appears more complex, the cost functions of the two protocols are the same. This is explained by the fact that, although TRQ may need to iterate, each iteration "consumes" a delta set that would have triggered an independent instance of IRQ. In practice, the actual cost of TRQ may be slightly higher than IRQ since TRQ must generate and communicate timestamps, and because restarts in TRQ may cause larger data sets to be transmitted. The cost of the MDS protocol can be "tuned" by varying the granularity factor $g$, i.e., the number of delta notifications before constraint checking is initiated. This allows an application to establish a constraint checking policy that balances its integrity requirements against the available resources. The efficiency of the LTT protocol depends heavily on the probability $p$ that a local test succeeds; if $p$ is low, the LTT protocol has higher cost than the TRT protocol. The actual value for $p$ is strongly application-dependent.

Our constraint checking protocols in Section 3 have been developed for constraints involving exactly two databases. It is our experience that two-site constraints predominate in practice. Furthermore, even when a constraint involves more than two databases, often it can be "split" into multiple constraints, each involving exactly two databases. There are, however, cases where we may need to handle multi-site constraints. Therefore, it may be necessary to extend our protocols to more than two sites.

Multi-site constraints can be evaluated easily using the DRQ or IRQ protocol - the constraint manager simply submits remote queries to the various sites and combines the results. Safety of the protocol is guaranteed by the same reasoning given in Section 3.1. Timestampbased protocols TRQ and SRQ also can be extended in a straightforward way to handle multi-site constraints: A restart is necessary whenever a busy status is received from any of the remote sites and a local update has occurred; safety and accuracy follow from the results in Section 3.2. It is less straightforward to construct safe and accurate versions of the transaction-based protocols (such as TRT) for the multi-site case. These protocols emulate two-phase locking, which requires coordinating local transactions across multiple sites. A two-phase "handshake" protocol could be used for synchronization, similar to distributed two-phase commit. Alternatively, a mechanism could be used where remote queries are "chained" from one site to the next (emulating multiple nested transactions). Unfortunately, both solutions incur a considerable loss of local autonomy. These initial observations lead us to believe that nontransaction-based protocols are most suitable for constraints involving more than two sites.

\section{Conclusions and Future Work}

We have described a family of cooperative constraint checking protocols for federated database systems. We have isolated two properties of constraint checking protocols that are of primary importance: safety and accuracy. All of our protocols are safe. Obtaining accuracy requires additional mechanisms, as seen in our more complex protocols. In addition to varying in terms of accuracy, our protocols also vary in terms of their requirements of the underlying systems, their level of asynchrony, their flexibility, and their execution costs. We are quite certain that no one protocol will be suitable for all federated database scenarios. By providing a family of alternatives, one protocol can be chosenand perhaps tailored-for a particular environment or application. By formalizing the relevant concepts, and by identifying and analyzing a suite of protocols, this paper provides a sound basis for the problem of integrity constraint checking in federated databases.

Our initial work can be elaborated in a number of directions. We plan to implement the protocols in a true loosely-coupled environment in order to measure 


\begin{tabular}{|c|c|c|c|c|}
\hline & local msgs per $\Delta$ & remote msgs per $\Delta$ & database ops per $\Delta$ & total cost (simplified) \\
\hline DRQ & $\lambda_{D}$ & $2 \rho$ & $\delta_{Q}$ & $(\lambda+2 \rho+\delta) \nu$ \\
IRQ & $3 \lambda_{D}$ & $2 \rho$ & $\delta_{Q}$ & $(3 \lambda+2 \rho+\delta) \nu$ \\
TRQ & $3 \lambda_{D}$ & $2 \rho$ & $\delta_{Q}$ & $(3 \lambda+2 \rho+\delta) \nu$ \\
SRQ & $3 \lambda_{D}$ & $2 \rho$ & $\delta_{Q}$ & $2(\lambda+\rho+\delta) \nu$ \\
TRT & $\lambda_{D}+\lambda_{N}$ & $2 \rho$ & $\delta_{Q}+\delta_{C}$ & $(\lambda+\delta+(3 \lambda+2 \rho+4 \delta) / g) \nu$ \\
MDS & $\lambda_{N}+\left(2 \lambda_{D}+\lambda_{N}\right) / g$ & $2 \rho / g$ & $\delta_{U}+\left(2 \delta_{Q}+\delta_{U}+\delta_{C}\right) / g$ & $(\lambda+\delta)$ \\
LTT & $3 \lambda_{D}+\lambda_{N}$ & $2(1-p) \rho$ & $(2-p) \delta_{Q}+\delta_{C}$ & $(4 \lambda+2(1-p) \rho+(3-p) \delta) \nu$ \\
\hline$\nu$ & number of update transactions & $\delta_{Q}$ & cost of local database query operation \\
$\lambda_{N}$ & cost of local notification-only message & $\delta_{U}$ & cost of local database update operation \\
$\lambda_{D}$ & cost of local data-conveying message & $\delta_{C}$ & cost of local database commit operation \\
$\rho$ & cost of remote message & $g$ & granularity factor for MDS \\
& & $p$ & probability of local test succes in LTT \\
\hline
\end{tabular}

Table 8: Cost functions for the protocols

and compare protocol performance on real systems. We intend to elaborate our model for analyzing the execution costs of the protocols by introducing and varying parameter values to understand relative costs in different environments. We plan to explore the issue of constraint repair when violations occur. Even in traditional centralized databases, constraint repair is an important topic of current research; in federated databases the problem is even more difficult. We plan to consider more carefully the protocols in the design space that we have not studied, to further investigate protocols for multi-site constraints, and to develop protocols for handling constraints that do not have a straightforward incremental form. Finally, we plan to investigate the use of logical clocks instead of absolute timestamps for those protocols relying on synchronized time services.

\section{Acknowledgments}

We are grateful to Stefano Ceri, Hector Garcia-Molina, and the rest of the Stanford Database Group for useful feedback on this work.

\section{References}

[1] D. Barbara and H. Garcia-Molina. The Demarcation Protocol: A technique for maintaining linear arithmetic constraints in distributed database systems. In $A d$ vances in Database Technology-EDBT' '92, LNCS 580. Springer-Verlag, Berlin, 1992.

[2] O. Babaoğlu and K. Marzullo. Consistent global states of distributed systems: Fundamental concepts and mechanisms. In S. Mullender, editor, Distributed Systems. ACM Press, New York, 1993.

[3] Y. Breitbart, H. Garcia-Molina, and A. Silberschatz. Overview of multidatabase transaction management. VLDB Journal, 1(2), 1992.

[4] S. Ceri and J. Widom. Managing semantic heterogeneity with production rules and persistent queues. In Procs. 19th Int. Conf. on Very Large Data Bases, Dublin, Ireland, 1993.

[5] S. Chawathe, H. Garcia-Molina, and J. Widom. A Toolkit for Constraint Management in Heterogeneous Information Systems. In Procs. 12th Int. Conf. on Data Engineering, New Orleans, LA, 1996.

[6] L. Do and P. Drew. Active database management of global data integrity constraints in heterogeneous database environments. In Procs. 11th Int. Conf. on Data Engineering, Taipei, Taiwan, 1995.
[7] P.W.P.J. Grefen and P.M.G. Apers. Parallel handling of integrity constraints on fragmented relations. In Procs. Int. Symp. on Databases in Parallel and Distributed Systems, Dublin, Ireland, 1990.

[8] P.W.P.J. Grefen and P.M.G. Apers. Integrity control in relational database systems - an overview. Journal of Data \& Knowledge Engineering, 10(2), 1993.

[9] P.W.P.J. Grefen. Combining theory and practice in integrity control: a declarative approach to the specification of a transaction modification subsystem. In Procs. 19th Int. Conf. on Very Large Data Bases, Dublin, Ireland, 1993.

[10] P. Grefen and J. Widom. Integrity constraint checking in federated databases. Memoranda Informatica, 94-80, CS Dept., University of Twente, 1994.

[11] A. Gupta, Y. Sagiv, J.D. Ullman, and J. Widom. Constraint checking with partial information. In Procs. 13 th ACM SIGACT-SIGMOD-SIGART Symp. on Principles of Database Systems, 1994.

[12] A. Gupta and J. Widom. Local verification of global integrity constraints in distributed databases. In Procs. ACM SIGMOD Int. Conf. on Management of Data, Washington, D.C., 1993.

[13] L. Lamport. Time, clocks, and the ordering of events in a distributed system. Comm. ACM, 21(7), 1978.

[14] D. Montesi, R.Torlone. A transaction transformation approach to active rule processing. In Procs. 11th Int. Conf. on Data Engineering, Taipei, Taiwan, 1995.

[15] X. Qian. Distribution design of integrity constraints. In Procs. 2nd Int. Conf. on Expert Database Systems, Benjamin/Cummings, Redwood City, California, 1989.

[16] X. Qian and G. Wiederhold. Incremental recomputation of active relational expressions. IEEE Trans. on Knowledge and Data Engineering, 3(3), 1991.

[17] M. Rusinkiewicz, A. Sheth, and G. Karabatis. Specifying interdatabase dependencies in a multidatabase environment. IEEE Computer, 24(12), 1991.

[18] E. Simon and P. Valduriez. Integrity control in distributed database systems. In Procs. 19th Int. Conf. on System Sciences, Hawaii, 1986.

[19] J. Widom and S. Ceri. Active Database Systems: Triggers and Rules for Advanced Database Processing. Morgan Kaufmann, San Francisco, California, 1996. 